\title{
Model-Free Synthesis of Fixed Structure Stabilizing Controllers Using the Rate of Change of Phase *
}

\author{
Navid Mohsenizadeh* Swaroop Darbha ${ }^{* *}$ Lee H. Keel ${ }^{* * *}$ \\ Shankar P. Bhattacharyya ${ }^{* * * *}$ \\ * Department of Mechanical Engineering, Texas A\& University, \\ College Station, TX 77843 USA (email: navidm@tamu.edu). \\ ** Department of Mechanical Engineering, Texas A\& M University, \\ College Station, TX 77843 USA (email: dswaroop@tamu.edu). \\ *** Department of Electrical and Computer Engineering, Tennessee \\ State University, Nashville, TN 37203 USA (e-mail: \\ keel@gauss.tsuniv.edu). \\ **** Department of Electrical Engineering, Texas A\& $M$ University, \\ College Station, TX 77843 USA (email: bhatt@ee.tamu.edu).
}

\begin{abstract}
This technical note outlines an approach to the problem of synthesizing fixedstructure low-order controllers for systems whose mathematical models are unknown, but their frequency responses are available. Stability of the closed-loop system is guaranteed if the frequency response of the plant and the controller satisfy certain conditions at specific frequencies. It is shown that the relation between the rate of change of phase of the plant and the controller, at specific frequencies, characterizes the closed-loop system stability. We use this characterization in a systematic way to design fixed-structure low-order controllers such as PI and PID-controllers.
\end{abstract}

Keywords: Bode plots, Nyquist stability criterion, Fixed-structure controllers.

\section{INTRODUCTION}

The problem of designing stabilizing controllers requires an accurate knowledge of the system. Most of the controller design techniques require a model of the system a priori. Modeling is an intermediate step in a design problem which intends to approximate the actual system with a simpler mathematical representation. In many fields of science and engineering one needs to deal with complex systems where modeling simplifications may result in unreliable models in the sense that the model loses the ability to capture all the actual system behaviours. A fatal consequence of such simplifications occurs when the designed controller stabilizes the model but not the actual system.

These observations motivate a new line of research to directly design controllers based on measurements. Some recent results on model-free design approaches are provided by Park and Ikeda (2004) and Yasumasa et al. (2005). Keel and Bhattacharyya (2008) proposed a method to design three term controllers based directly on frequency domain test data. A Bode plot characterization of all stabilizing controllers is presented by Keel and Bhattacharyya (2010). They showed that if the frequency response of the plant and the controller satisfy certain conditions at specific frequencies, the closed-loop system is stable. These conditions depend on the rate of change of phase of the plant

\footnotetext{
* This work was supported by National Science Foundation grant numbers CMMI-0927652 and CMMI-0927664.
}

and the controller. In this paper we use these conditions to develop a systematic method in designing fixed-structure controllers based on the frequency response measurements.

Since in our proposed approach the structure of the controller is pre-assumed, the design problem can be handled for low-order simple controllers such as PID-controllers. Recent developments in designing PID-controllers are presented by Bhattacharyya et al. (2009). Low-order controllers are of interest in practical applications because of their simple structure, easy manufacturing and maintenance.

This paper is organized as follows: In section 2 we present our main results for different classes of controllers, such as PI and PID-controllers. For each class of controllers we illustrate the proposed method using an example. Section 3 contains some concluding remarks.

\section{MAIN RESULTS}

In this section we present the main results of the paper for 4 classes of fixed-structure low-order controllers and provide illustrative examples to show how the method developed here can be used to solve a controller synthesis problem.

\subsection{Constant-gain controller: $C(s)=k$}

The first class of controllers being studied is constantgain controllers, $C(s)=k$. The necessary and sufficient 
conditions under which this class of controllers stabilizes a general plant, where only the frequency response of the plant is available, are established. In the following, the required preliminaries are provided first and then the stability conditions are developed.

Case 1) $k \geq 0$ :

Let $P(j \omega)$ be the frequency response of a plant with no $j \omega$ poles, and denote by $\Omega_{+}=\left\{\omega_{1}, \omega_{2}, \ldots, \omega_{l}\right\}$, the sequence of the roots of

$$
\angle P^{-1}(j \omega) \pm n \pi=0, n=1,3,5, \ldots,
$$

where

$$
\left|P^{-1}(j \omega)\right|_{\omega=\omega_{1}}<\left|P^{-1}(j \omega)\right|_{\omega=\omega_{2}}<\ldots<\left|P^{-1}(j \omega)\right|_{\omega=\omega_{l}} .
$$

For each root, $\omega_{t}$, of (1), an integer, $i_{t}$, may be associated based on the rate of change of phase of $P^{-1}(j \omega)$, as follows:

$$
i_{t}=\left\{\begin{array}{l}
+2 \text { if }\left.\frac{d}{d \omega} \angle P^{-1}(j \omega)\right|_{\omega=\omega_{t}}<0 \\
0 \text { if }\left.\frac{d}{d \omega} \angle P^{-1}(j \omega)\right|_{\omega=\omega_{t}}=0 \\
-2 \text { if }\left.\frac{d}{d \omega} \angle P^{-1}(j \omega)\right|_{\omega=\omega_{t}}>0
\end{array}\right.
$$

and if $\omega=0$ and/or $\omega=\infty$ are the roots of (1), then the corresponding integer(s) may be obtained from:

$$
i_{t}=\left\{\begin{array}{l}
+1 \text { if }\left.\frac{d}{d \omega} \angle P^{-1}(j \omega)\right|_{\omega=\omega_{t}=0} \text { or } \infty<0 \\
0 \quad \text { if }\left.\frac{d}{d \omega} \angle P^{-1}(j \omega)\right|_{\omega=\omega_{t}=0} \text { or } \infty=0 \\
-1 \text { if }\left.\frac{d}{d \omega} \angle P^{-1}(j \omega)\right|_{\omega=\omega_{t}=0} \text { or } \infty>0 .
\end{array}\right.
$$

Therefore, corresponding to the sequence of frequencies $\Omega_{+}=\left\{\omega_{1}, \omega_{2}, \ldots, \omega_{l}\right\}$, one may associate a sequence of integers $I_{+}=\left\{i_{1}, i_{2}, \ldots, i_{l}\right\}$.

Case 2) $k<0$ :

In this case one may consider the frequency response of $-P(j \omega)$, and solve the following set of equations:

$$
\angle-P^{-1}(j \omega) \pm n \pi=0, n=1,3,5, \ldots,
$$

to find the sequence of frequencies $\Omega_{-}$. The corresponding sequence of integers $I_{-}$may be calculated using (2) and $(3)$, using the frequency response of $-P^{-1}(j \omega)$.

If the plant has pole(s) at the origin and/or has $j \omega$ poles, the corresponding integers can be obtained using the results presented by Keel and Bhattacharyya (2010).

Now, we state the conditions for constant-gain stabilizability of the feedback control system shown in Fig. 1.

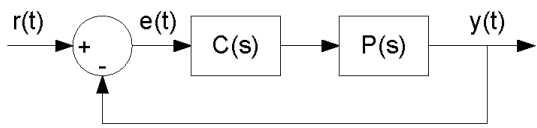

Fig. 1. Schematic of a continuous-time unity feedback control system

Theorem 1. The unity feedback control system shown in Fig. 1 is constant-gain stabilizable if and only if

$$
\max _{m} \sum_{t=1}^{m} i_{t}=p^{+}, \quad m=1,2, \ldots, l, i \in I
$$

where $p^{+}$is the number of RHP poles of the plant and I is the sequence of integers, $I_{+}$or $I_{-}$, introduced earlier.
Furthermore, for any $m^{*}$ that $\sum_{t=1}^{m^{*}} i_{t}=p^{+}$and $i \in I_{+}$, then the interval

$$
k \in\left[\left|P^{-1}(j \omega)\right|_{\omega=\omega_{m *}},\left|P^{-1}(j \omega)\right|_{\omega=\omega_{m *+1}}\right]
$$

is in the stability set for $k \geq 0$, and for any $m^{*}$ that $\sum_{t=1}^{m^{*}} i_{t}=p^{+}$and $i \in I_{-}$, then the interval

$$
k \in\left[-\left|P^{-1}(j \omega)\right|_{\omega=\omega_{m^{*}+1}},-\left|P^{-1}(j \omega)\right|_{\omega=\omega_{m^{*}}}\right]
$$

is in the stability set for $k<0$.

Proof. Proof of this theorem is constructed based on the results developed by Keel and Bhattacharyya (2010). Consider the following two cases:

Case 1) $k \geq 0$ :

Let us denote the magnitude of $P^{-1}(j \omega)$ at the frequency $\omega_{t}$ by $M_{t}=\left|P^{-1}(j \omega)\right|_{\omega=\omega_{t}}$. Now, consider all the roots of $\angle P^{-1}(j \omega) \pm n \pi=0, n=1,3,5, \ldots$, and denote them by $\Omega_{+}=\left\{\omega_{1}, \omega_{2}, \ldots, \omega_{l}\right\}$, such that

$$
M_{1}<M_{2}<\ldots<M_{l} \text {. }
$$

Assume that $k=k^{*} \geq 0$, and denote the roots of the following set of equations

$$
\begin{aligned}
& \angle P^{-1}(j \omega) \pm n \pi=0, \quad n=1,3,5, \ldots, \\
& \left|P^{-1}(j \omega)\right|<k^{*}
\end{aligned}
$$

by $\Omega_{+}^{*}=\left\{\omega_{1}, \omega_{2}, \ldots, \omega_{m}\right\}$. It is clear that the solution set of (5) does not change for $k^{*} \in\left[M_{m}, M_{m+1}\right]$. For this interval of constant gain values, the controller $C(s)=k^{*}$ stabilizes the plant provided that

$$
i(G):=\sum_{t=1}^{m} i_{t}=p^{+},
$$

where $i_{t}$ is as defined previously. Therefore, for each interval $\left[M_{t}, M_{t+1}\right], t=0,1, \ldots, l$, where $M_{0}=0$ and $M_{l+1}=\infty$, one may check if the equality $\sum_{t} i_{t}=p^{+}$holds for stability. It is clear that the most possible stabilizing interval is the one for which $\sum_{t} i_{t}$ is maximum.

Case 2) $k<0$ :

In order to consider the negative gain values, i.e. $k<0$, one may replace $P^{-1}(j \omega)$ by $-P^{-1}(j \omega)$, and follow the same procedure. The corresponding stability intervals obtained from this case should be finally multiplied by -1 . This completes the proof.

Example 1. Consider the following frequency response of an unknown plant which has no RPH poles; i.e. $p^{+}=0$.
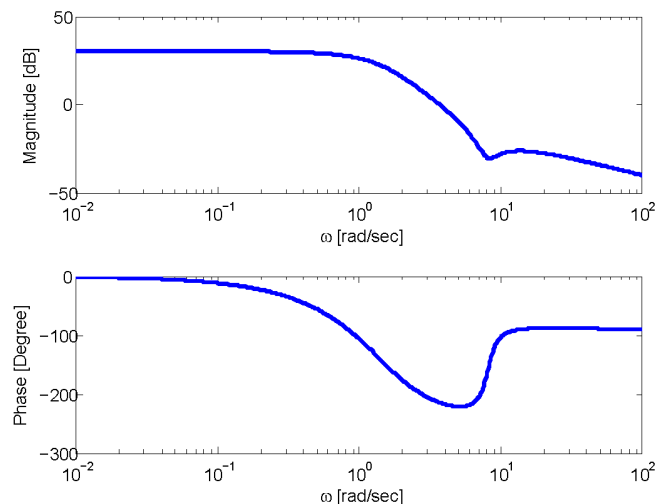

Fig. 2. Frequency response of an unknown plant 
Now, the synthesis problem is to determine the set of all constant-gain controllers which stabilizes the closed-loop system shown in Fig.1.

We consider two cases, $k \geq 0$ and $k<0$.

Case 1) $k \geq 0$ :

First, one needs to solve the following set of equations:

$$
\angle P^{-1}(j \omega) \pm n \pi=0, n=1,3,5, \ldots
$$

As shown in Fig.3, (6) has 2 roots, $\Omega_{+}=\left\{\omega_{1}=2.1, \omega_{2}=\right.$ $7.7\}$. The magnitudes of $P^{-1}(j \omega)$ at these frequencies are $M_{+}=\left\{M_{1}=0.18, M_{2}=27.3\right\}$, respectively. Note that, as required by theorem $1, M_{1}<M_{2}$. Now, one may find the corresponding sequence of integers as $I_{+}=\left\{i_{1}=-2, i_{2}=\right.$ $+2\}$ using (2) and the last graph of Fig.3. The intervals to be considered for this case are:

$$
I_{1}=[0,0.18), I_{2}=[0.18,27.3), \quad I_{3}=[27.3, \infty) .
$$

The corresponding $i(G)$ for each interval is:

$$
\begin{aligned}
& i(G)_{I_{1}}=0=p^{+}, \\
& i(G)_{I_{2}}=i_{1}=-2<p^{+}, \\
& i(G)_{I_{3}}=i_{1}+i_{2}=-2+2=0=p^{+} .
\end{aligned}
$$

Thus, the stability intervals for the case $k \geq 0$ are:

$$
\mathbb{S}_{+}=[0,0.18) \cup[27.3, \infty)
$$

Case 2) $k<0$ :

For this case we have:

$$
\angle-P^{-1}(j \omega) \pm n \pi=0, n=1,3,5, \ldots
$$

which has only one root at $\Omega_{-}=\left\{\omega_{1}=0\right\}$ for which the magnitude of $-P^{-1}(j \omega)$ is $M_{-}=\left\{M_{1}=0.03\right\}$. Now, one may find the corresponding sequence of integer as $I_{-}=\left\{i_{1}=-1\right\}$. The intervals to be considered for this case are:

$$
I_{1}=[0,0.03), I_{2}=[0.03, \infty) .
$$

The corresponding $i(G)$ for each interval is:

$$
\begin{aligned}
& i(G)_{I_{1}}=0=p^{+}, \\
& i(G)_{I_{2}}=i_{1}=-1<p^{+} .
\end{aligned}
$$

The interval $[0,0.03)$ stabilizes the plant $-P(j \omega)$; equivalently, the interval $(-0.03,0]$ stabilizes the original plant $P(j \omega)$. Thus, the stability interval for the case $k<0$ is:

$$
\mathbb{S}_{-}=(-0.03,0]
$$

The union of the stability sets $\mathbb{S}_{+}$and $\mathbb{S}_{-}$will be the entire stability set, which is:

$$
\mathbb{S}=\mathbb{S}_{+} \cup \mathbb{S}_{-}=[-0.03,0.18] \cup[27.3, \infty) .
$$

\subsection{Integrator controller: $C(s)=k / s$}

In this subsection we consider an integrator controller $C(s)=k / s$ to stabilizes the closed-loop system shown in Fig.1. Let $P(j \omega)$ be the frequency response of an unknown plant and denote by $\Omega_{+}=\left\{\omega_{1}, \omega_{2}, \ldots, \omega_{l}\right\}$, the roots of

$$
\angle P^{-1}(j \omega) \pm n \pi=-\pi / 2, n=1,3,5, \ldots
$$

where

$$
\begin{aligned}
& \left|\omega P^{-1}(j \omega)\right|_{\omega=\omega_{1}}<\left|\omega P^{-1}(j \omega)\right|_{\omega=\omega_{2}}<\ldots \\
& \ldots<\left|\omega P^{-1}(j \omega)\right|_{\omega=\omega_{l}} .
\end{aligned}
$$

For each $\omega_{t}$ satisfying (7) and (8), one may associate an integer as explained in the previous section. In this case,
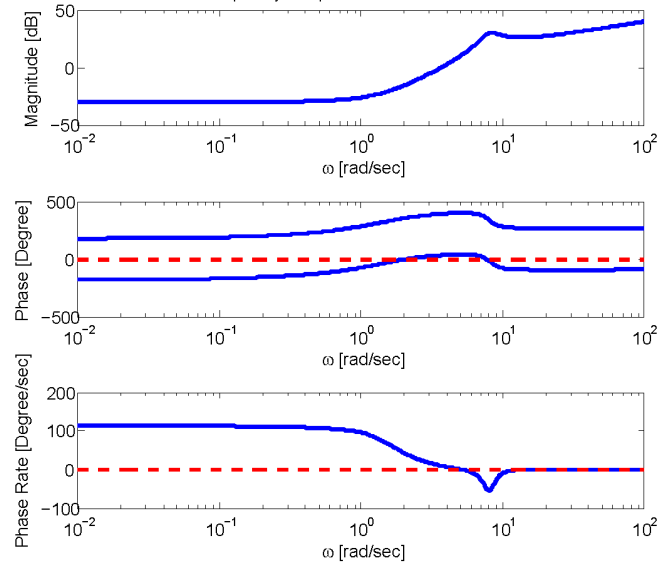

Fig. 3. Frequency response of the inverse plant

it is possible to have a nyquist plot cut to the left of the point $-1+0 j$ at $\omega=0$. Thus, the sequence of frequencies $\Omega$ always includes $\omega=0$. If the plant has no pole at the origin, the integer corresponding to $\omega=0$ can be obtained from:

$$
i= \begin{cases}0 & \text { if } P\left(0^{+}\right) C\left(0^{+}\right)>0 \\ -1 & \text { if } P\left(0^{+}\right) C\left(0^{+}\right)<0 .\end{cases}
$$

If the plants has pole(s) at the origin, one may use the formulas provided by Keel and Bhattacharyya (2010), instead of (9), to obtain the corresponding integer.

Theorem 2. The unity feedback control system shown in Fig. 1 is $\frac{k}{s}$-stabilizable if and only if

$$
\max _{m} \sum_{t=1}^{m} i_{t}=p^{+}, \quad m=1,2, \ldots, l, i \in I
$$

where $p^{+}$is the number of RHP poles of the plant and I is the sequence of integers, $I_{+}$or $I_{-}$, introduced earlier. Furthermore, for any $m^{*}$ that $\sum_{t=1}^{m^{*}} i_{t}=p^{+}$and $i \in I_{+}$, then the interval

$$
k \in\left[\left|\omega P^{-1}(j \omega)\right|_{\omega=\omega_{m^{*}}},\left|\omega P^{-1}(j \omega)\right|_{\omega=\omega_{m^{*}+1}}\right]
$$

is in the stability set for $k \geq 0$, and for any $m^{*}$ that $\sum_{t=1}^{m^{*}} i_{t}=p^{+}$and $i \in I_{-}$, then the interval

$$
k \in\left[-\left|\omega P^{-1}(j \omega)\right|_{\omega=\omega_{m^{*}+1}},-\left|\omega P^{-1}(j \omega)\right|_{\omega=\omega_{m^{*}}}\right]
$$
is in the stability set for $k<0$.

Proof. Proof of this theorem is the same as the proof of theorem 1; however, the controller introduces a pole at the origin in the forward loop gain $G(s)=P(s) C(s)$ which its corresponding nyquist plot cut should be considered separately at $\omega=0$.

Example 2. Consider the frequency response of the unknown plant shown in Fig.2 again. In this example we attempt to stabilize the closed-loop system using an integrator controller, $C(s)=k / s$

First, consider $\omega_{1}=0$ for which $M_{1}=0$, and $i_{1}=0$ since $P\left(0^{+}\right) C\left(0^{+}\right)>0$ and the plant has no pole at the origin. Similar to the previous example, we consider the following two cases:

Case 1) $k \geq 0$ :

The following set of equations 


$$
\angle P^{-1}(j \omega) \pm n \pi=-\pi / 2, n=1,3,5, \ldots
$$

has 2 roots, $\omega_{2}=0.83, \omega_{3}=13.6$. The magnitudes of $\omega P^{-1}(j \omega)$ at these frequencies are $M_{2}=0.03, M_{3}=279$. Thus we have $\Omega_{+}=\left\{\omega_{1}, \omega_{2}, \omega_{3}\right\}$ for which, as required by theorem $2, M_{1}<M_{2}<M_{3}$. The corresponding sequence of integers is $I_{+}=\left\{i_{1}=0, i_{2}=-2, i_{3}=+2\right\}$. The intervals to be considered for this case are:

$$
I_{1}=[0,0.03), \quad I_{2}=[0.03,279), \quad I_{3}=[279, \infty) .
$$

The corresponding $i(G)$ for each interval is:

$$
\begin{aligned}
& i(G)_{I_{1}}=i_{1}=0=p^{+}, \\
& i(G)_{I_{2}}=i_{1}+i_{2}=-2<p^{+}, \\
& i(G)_{I_{3}}=i_{1}+i_{2}+i_{3}=0=p^{+} .
\end{aligned}
$$

Thus, the stability intervals for the case $k \geq 0$ are:

$$
\mathbb{S}_{+}=[0,0.03) \cup[279, \infty) .
$$

Case 2) $k<0$ :

In this case for $\omega_{1}=0$ we have $i_{1}=-1$ because $P\left(0^{+}\right) C\left(0^{+}\right)<0$. Also the set of equations

$$
\angle-P^{-1}(j \omega) \pm n \pi=-\pi / 2, n=1,3,5, \ldots
$$

has no root. Therefore, for the interval $k<0$ we have $i(G)=-1<p^{+}$, which implies that $k<0$ is not in the stability set, or $\mathbb{S}_{-}=\emptyset$. The entire stability set is the union of the intervals from the two cases, which is:

$$
\mathbb{S}=\mathbb{S}_{+} \cup \mathbb{S}_{-}=[0,0.03] \cup[279, \infty) \text {. }
$$

\subsection{PI-controller: $C(s)=k_{p}+k_{i} / s$}

In this subsection we focus on PI-controllers and derive the conditions under which this class of controllers stabilizes the closed-loop system shown in Fig.1. The controller transfer function can be written as $C(s)=\frac{k}{s}(T s+1)$. Let us assume a new plant $\bar{P}(s)=(T s+1) P(s)$ which should be stabilizable by an integrator controller $\bar{C}(s)=k / s$. In this case, $(7)$ can be modified as:

which is

$$
\angle \bar{P}^{-1}(j \omega) \pm n \pi=-\pi / 2, n=1,3,5, \ldots
$$

$$
\angle P^{-1}(j \omega)-\arctan (T \omega) \pm n \pi=-\pi / 2, n=1,3,5, \ldots
$$

or

$$
\angle P^{-1}(j \omega)+\pi / 2 \pm n \pi=\arctan (T \omega), n=1,3,5, \ldots
$$

The left-hand side of (12) is known while the right-hand side can be plotted as a family of curves for acceptable intervals of $T$ which gives enough number of roots required to satisfy the stability condition stated in Theorem 2. Let us choose a value of $T^{*}$ and denote the roots of

$$
\angle P^{-1}(j \omega)+\pi / 2 \pm n \pi=\arctan \left(T^{*} \omega\right), n=1,3,5, \ldots
$$

by $\Omega_{+}=\left\{\omega_{1}, \omega_{2}, \ldots, \omega_{l}\right\}$ where

$$
\begin{aligned}
& \left|\omega \bar{P}^{-1}(j \omega)\right|_{\omega=\omega_{1}}<\left|\omega \bar{P}^{-1}(j \omega)\right|_{\omega=\omega_{2}}<\ldots \\
& \ldots<\left|\omega \bar{P}^{-1}(j \omega)\right|_{\omega=\omega_{l}} .
\end{aligned}
$$

Note that $\omega=0$ is included in the sequence of frequencies $\Omega_{+}$. The rate of change of phase of $\bar{P}^{-1}(j \omega)$, assuming $T=T^{*}$, can be calculated as:

$$
\frac{d}{d \omega} \angle \bar{P}^{-1}(j \omega)=\frac{d}{d \omega} \angle P^{-1}(j \omega)-\frac{d}{d \omega} \arctan \left(T^{*} \omega\right),
$$

or

$$
\frac{d}{d \omega} \angle \bar{P}^{-1}(j \omega)=\underbrace{\frac{d}{d \omega} \angle P^{-1}(j \omega)}_{\text {known }}-\underbrace{\frac{T^{*}}{1+\omega^{2} T^{* 2}}}_{\text {known }} .
$$

The sequence of integers can be obtained as explained earlier. Thus, at each $T^{*}$, one may find stability intervals $\mathbb{S}_{+\left(T=T^{*}\right)}$ for the gain $k$, if they exist. For negative gains, i.e. $k<0$, one may substitute $\bar{P}^{-1}(j \omega)$ by $-\bar{P}^{-1}(j \omega)$ in (11), assuming $T=T^{*}$, to find the corresponding sequences $\Omega_{-}$and $I_{-}$and determine the stability intervals $\mathbb{S}_{-\left(T=T^{*}\right)}$ for $k<0$, if they exist. The stability intervals at $T=T^{*}$ will be $\mathbb{S}_{\left(T=T^{*}\right)}=\mathbb{S}_{+\left(T=T^{*}\right)} \cup \mathbb{S}_{-\left(T=T^{*}\right)}$. By sweeping the values of $T^{*}$ over an admissible range, one can construct the entire stability set, $\mathbb{S}$, in the space of the controller parameters $(T, k)$.

Example 3. In this example we explain how to obtain the set of all PI-controllers stabilizing an unknown plant using the approach presented here. Consider the frequency response of an unknown plant with one RHP pole, i.e. $p^{+}=1$, shown in Fig. 4 .
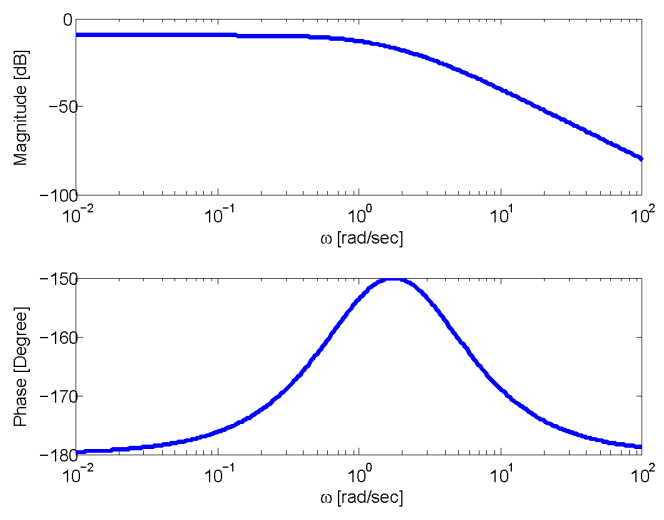

Fig. 4. Frequency response of an unknown plant

A family of curves can be obtained for a range of $T$ values in (12). Let us choose $T^{*}=1$ from this family of curves and further our procedure for the following two cases:

Case 1) $k \geq 0$ :

At $\omega_{1}=0$ we have $\bar{P}\left(0^{+}\right) \bar{C}\left(0^{+}\right)<0$ and thus $i_{1}=-1$. Solving (13) (Fig.5), the frequency sequence will be $\Omega_{+}=$ $\left\{\omega_{1}=0, \omega_{2}=\sqrt{3}\right\}$. At $\omega_{2}=\sqrt{3}$ the rate of change of phase of $\bar{P}(j \omega)$ is negative, using (15), and thus $i_{1}=+2$. The sequence of integers for this case will be $I_{+}=\{-1,+2\}$. Note that the magnitudes of $\omega \bar{P}^{-1}(j \omega)$ at $\omega_{1}$ and $\omega_{2}$ are $M_{1}=0$ and $M_{2}=6$, respectively; for which, as required by theorem $2, M_{1}<M_{2}$. The intervals to be considered for $k \geq 0$ are:

$$
I_{1}=[0,6), I_{2}=[6, \infty) .
$$

The corresponding $i(G)$ for each interval is:

$$
\begin{aligned}
& i(G)_{I_{1}}=-1<p^{+}, \\
& i(G)_{I_{2}}=-1+2=1=p^{+}
\end{aligned}
$$

Thus, the stability interval for $k \geq 0$ at $T=1$ is $\mathbb{S}_{+(T=1)}=[6, \infty)$.

Case 2) $k<0$ :

In this case for $\omega_{1}=0$ we have $i_{1}=0$ because 
$\bar{P}\left(0^{+}\right) \bar{C}\left(0^{+}\right)>0$. Also the set of equations (13) have no root. Therefore, for the interval $k<0$ we have $i(G)=0<$ $p^{+}$, which implies that $k<0$ is not in the stability set, or $\mathbb{S}_{-(T=1)}=\emptyset$.

The entire stability set at $T=1$ is the union of the intervals from the two cases, which is:

$$
\mathbb{S}_{(T=1)}=\mathbb{S}_{+(T=1)} \cup \mathbb{S}_{-(T=1)}=[6, \infty) .
$$

Fig. 6 shows the entire stability region in the space of the controller parameters $(T, k)$ obtained from Routh-Hurwitz stability inequalities. As can be seen, at $T=1$, the stability interval for $k$ is $[6, \infty)$.
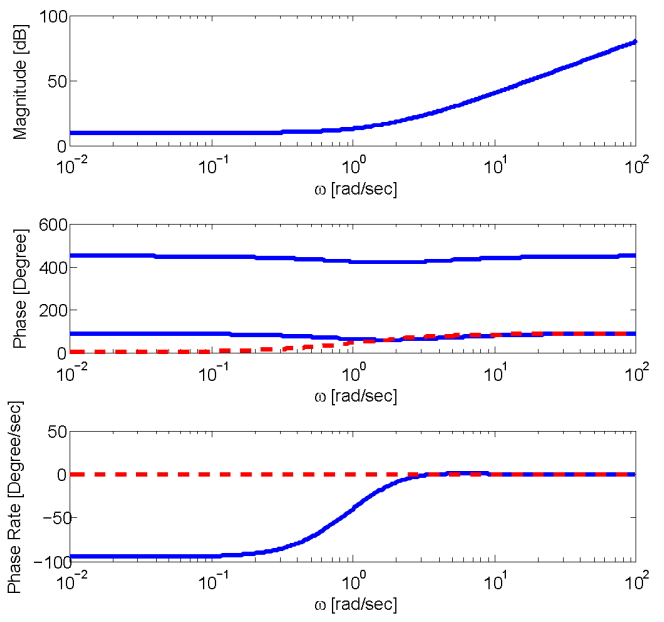

Fig. 5. Frequency response of the inverse plant and the controller

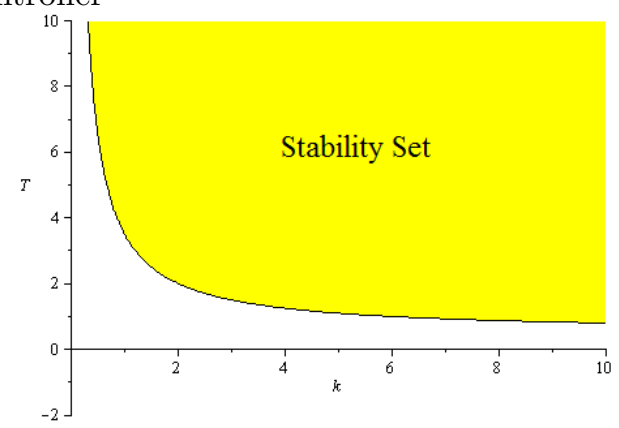

Fig. 6. Stability set for example 3

\subsection{PID-controller: $C(s)=k_{p}+k_{i} / s+k_{d} s$}

In this subsection we extend our method and consider PIDcontrollers to stabilize the closed-loop system shown in Fig.1. We write the controller transfer function as $C(s)=$ $\frac{k}{s}\left(T_{1} s+1\right)\left(T_{2} s+1\right)$ and define a new plant $\bar{P}(s)=\left(T_{1} s+\right.$ 1) $\left(T_{2} s+1\right) P(s)$ which for stability should be stabilizable by an integrator controller $\bar{C}(s)=k / s$. Following the same approach, one may write

which is

$$
\angle \bar{P}^{-1}(j \omega) \pm n \pi=-\pi / 2, n=1,3,5, \ldots,
$$

$$
\begin{aligned}
& \angle P^{-1}(j \omega)-\arctan \left(T_{1} \omega\right)-\arctan \left(T_{2} \omega\right) \pm n \pi=-\pi / 2, \\
& n=1,3,5, \ldots . \\
& \text { or } \\
& \quad \angle P^{-1}(j \omega)+\pi / 2 \pm n \pi=\arctan \left(T_{1} \omega\right)+\arctan \left(T_{2} \omega\right), \\
& n=1,3,5, \ldots .
\end{aligned}
$$

The left-hand side of (16) is known; however, the righthand side can be plotted as a family of curves for admissible intervals of $T_{1}$ and $T_{2}$ which yields enough number of roots required for stability as stated in Theorem 2 . Choosing values of $T_{1}^{*}$ and $T_{2}^{*}$, one may follow the same approach described in the previous subsection to find stability intervals for the gain $k$, if they exist.

Example 4. This example illustrates how the set of all stabilizing PID-controllers can be obtained for an unknown plant for which only the frequency response is available. Consider the frequency response of an unknown plant shown in Fig. 7 with two RHP poles, i.e. $p^{+}=2$.
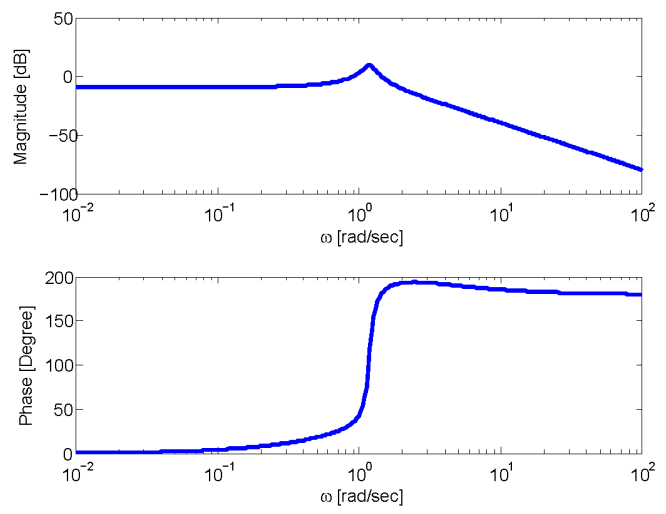

Fig. 7. Frequency response of an unknown plant

A family of curves can be obtained for different values of $T_{1}$ and $T_{2}$ in (16). Let us choose $T_{1}^{*}=1$ and $T_{2}^{*}=5$ from this family of curves and consider the following two cases:

Case 1) $k \geq 0$ :

Since at $\omega_{1}=0$ we have $\bar{P}\left(0^{+}\right) \bar{C}\left(0^{+}\right)>0$, the corresponding integer will be $i_{1}=-1$. Substituting the values of $T_{1}^{*}$ and $T_{2}^{*}$ into (16) and finding the roots (Fig.8), the frequency sequence will be $\Omega_{+}=\left\{\omega_{1}=0, \omega_{2}=1.2\right\}$. At $\omega_{2}=1.2$ the rate of change of phase of $\bar{P}(j \omega)$ is negative (Fig.8), and thus $i_{1}=+2$. The sequence of integers for this case will be $I_{+}=\{0,+2\}$. Note that the magnitudes of $\omega \bar{P}^{-1}(j \omega)$ at $\omega_{1}$ and $\omega_{2}$ are $M_{1}=0$ and $M_{2}=0.05$, respectively; for which, as required by theorem $2, M_{1}<M_{2}$. The intervals to be considered for $k \geq 0$ are:

$$
I_{1}=[0,0.05), \quad I_{2}=[0.05, \infty) .
$$

The corresponding $i(G)$ for each interval is:

$$
\begin{aligned}
& i(G)_{I_{1}}=0<p^{+}, \\
& i(G)_{I_{2}}=0+2=p^{+},
\end{aligned}
$$

Thus, the stability interval for $k \geq 0$ at $T_{1}^{*}=1$ and $T_{2}^{*}=5$ is $\mathbb{S}_{+\left(T_{1}^{*}=1, T_{2}^{*}=5\right)}=[0.05, \infty)$.

Case 2) $k<0$ :

In this case for $\omega_{1}=0$ we have $i_{1}=-1$ because $\bar{P}\left(0^{+}\right) \bar{C}\left(0^{+}\right)<0$ and the plant has no pole at the origin. Also the set of equations (16) has one root at $\omega_{2}=$ 0.34 (Fig.9). The corresponding sequences can be easily obtained as $\Omega_{-}=\left\{\omega_{1}=0, \omega_{2}=0.34\right\}, I_{+}=\left\{i_{1}=\right.$ $\left.-1, i_{2}=2\right\}$ and $M_{-}=\left\{M_{1}=0, M_{2}=0.43\right\}$ (Fig.9). The intervals to be considered here are:

$$
I_{1}=[0,0.43), \quad I_{2}=[0.43, \infty) .
$$

The corresponding $i(G)$ for each interval is: 

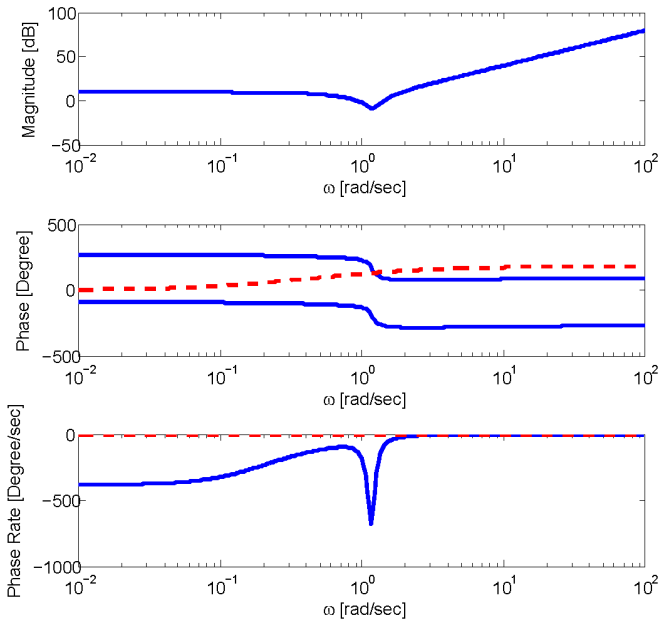

Fig. 8. Frequency response of the inverse plant and the controller (for $k \geq 0$ )

$$
\begin{aligned}
& i(G)_{I_{1}}=-1<p^{+}, \\
& i(G)_{I_{2}}=-1+2=1<p^{+},
\end{aligned}
$$

Therefore, for the interval $k<0$ we always have $i(G)=$ $0<p^{+}$, which implies that $k<0$ is not in the stability set, or $\mathbb{S}_{-\left(T_{1}^{*}=1, T_{2}^{*}=5\right)}=\emptyset$.

The entire stability set at $T_{1}=1, T_{2}=5$ is the union of the intervals from the two cases, which is:

$$
\mathbb{S}_{\left(T_{1}^{*}=1, T_{2}^{*}=5\right)}=[0.05, \infty) \text {. }
$$
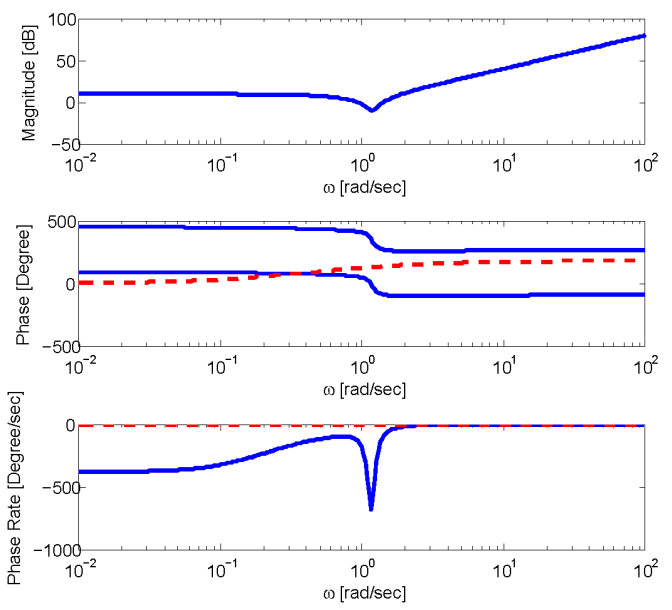

Fig. 9. Frequency response of the inverse plant and the controller (for $k<0$ )

\section{CONCLUDING REMARKS}

In this paper we developed a method to synthesize the set of all stabilizing fixed-structure low-order controllers for the systems whose frequency responses are available. This measurement-based approach does not require a mathematical model of the system which means that the synthesis problem can be carried out directly from the frequency response measurements. We showed how the rate of change of phase plays an important role in the controller design problem.

\section{REFERENCES}

Bhattacharyya, S.P., Datta, A., and Keel, L.H. (2009). Linear Control Theory: Structure, Robustness, and Optimization. CRC Press, Boca Raton, FL.

Keel, L.H. and Bhattacharyya, S.P. (2008). Controller synthesis free of analytical models: Three term controllers. IEEE Transactions on Automatic Control, 53(6), 13531369.

Keel, L.H. and Bhattacharyya, S.P. (2010). A bode plot characterization of all stabilizing controllers. IEEE Transactions on Automatic Control, 55(11), 2650-2654.

Park, U.S. and Ikeda, M. (2004). Data-based stability analysis for linear discrete-time system. In $43 \mathrm{rd}$ IEEE Conf. Decision Control, 1721-1723.

Yasumasa, F., Duan, Y., and Ikeda, M. (2005). System representation and optimal tracking in data space. In 16th IFAC World Congress. 